\title{
On Mott-Schottky analysis interpretation of capacitance measurements in organometal perovskite solar cells
}

Article in Applied Physics Letters · October 2016

DOI: $10.1063 / 1.4966127$

\section{CITATIONS}

0

4 authors, including:

Osbel Almora Rodríguez

Universitat Jaume I

10 PUBLICATIONS 96 CITATIONS

SEE PROFILE

\section{Germà Garcia-Belmonte}

Universitat Jaume I

195 PUBLICATIONS 7,274 CITATIONS

SEE PROFILE
READS

94

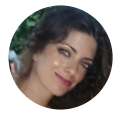

Clara Aranda

Universitat Jaume I

4 PUBLICATIONS 28 CITATIONS

SEE PROFILE

Some of the authors of this publication are also working on these related projects:

Project Perovskite materials for energy devices. View project 


\title{
On Mott-Schottky analysis interpretation of capacitance measurements in organometal perovskite solar cells
}

Osbel Almora, Clara Aranda, Elena Mas-Marza and Germà Garcia-Belmonte* Institute of Advanced Materials (INAM), Universitat Jaume I, 12006 Castelló, Spain

\begin{abstract}
\end{abstract}
Capacitance response of perovskite-based solar cells (PSCs) can be exploited to infer underlying physical mechanisms, both in the materials bulk and at outer interfaces. Particularly interesting is applying the depletion layer capacitance theory to PSCs, following common procedures used with inorganic and organic photovoltaic devices. Voltage-modulation of the depletion layer width allows extracting relevant parameters as the absorber defect density and built-in potential by means of the Mott-Schottky (MS) analysis. However, the uncritical use of the MS technique may be misleading and yields incorrect outcomes as a consequence of masking effects that accumulation capacitances, commonly observed in PSCs, produce on the measured capacitance value. Rules are provided here to select the measuring frequency that allows extracting depletion layer capacitance, and the voltage range in which it dominates, avoiding accumulation capacitive parasitic contributions. It is noted that the distinction of the depletion capacitance from the accumulation capacitance is only feasible in the case of perovskite layers containing significant defect density $\left(\sim 10^{17} \mathrm{~cm}^{-3}\right)$. It is confirmed that MS reproducibility is assured by hysteresis reduction at slow scan rates, and positive bias starting polarization. A complete procedure with specific checking points is provided here for consistent MS measurement and interpretation.

*Corresponding author: G. Garcia-Belmonte, (garciag@uji.es) tel.: +34 964387538

10 October 2016 
Since the successful introduction of hybrid perovskite compounds as $\mathrm{CH}_{3} \mathrm{NH}_{3} \mathrm{PbI}_{3}$ $\left(\mathrm{MAPbI}_{3}\right)$, acting as light absorber materials (in 2012 all-solid-state solar cells with 10 $\%$ power conversion efficiency were obtained $)^{1,2}$ there have been an unprecedented development of the so called Perovskite Solar Cells (PSCs) reaching performances as high as $22.1 \%$. ${ }^{3}$ These devices basically consist of a layered architecture where the absorber perovskite is sandwiched between an electron transport material (ETM), regularly $\mathrm{TiO}_{2}$, and a hole transport material (HTM), usually made of the organic compound 2,2',7,7'-tetrakis(N,N-di-p-methoxyphenylamine)-9,9-spirobifluorene (spiroOMeTAD). ${ }^{4,5}$ Recently significant attention has been paid to the capacitive response of this kind of photovoltaic device. ${ }^{6}$ The observation of a huge capacitance in the low frequency region of the spectrum, and its variation with the light intensity, have been interpreted in terms of contact or electrode charge accumulation mechanisms. ${ }^{7}$ Other types of solar technologies also exhibit capacitive responses related to contact phenomena as the depletion layer capacitance commonly exhibited by inorganic and organic photovoltaic devices. ${ }^{8}$ Voltage-modulation of the depletion layer width $(w)$ allows extracting relevant parameters as the absorber acceptor defect density $(N)$ and built-in potential $\left(V_{b i}\right)$ by means of capacitance-voltage $(C-V)$ Mott-Schottky (MS) analysis. ${ }^{9}$ These two parameters can be used to derive experimentally-supported PSCs device models, and also constitute key information of variations in materials properties induced by device processing modifications. However, the direct application of the MS technique to PSCs is challenging because of the masking effects of additional (and large) accumulation capacitance contributions. ${ }^{10-19}$ In addition, there is still a lot of research and debate around several current distorting behaviors producing hysteresis effects, ${ }^{6,20,21}$ whose implications are often unnoticed when analyzing typical MS plots. Therefore, the uncritical use of the MS technique may be misleading and yields incorrect outcomes. In this letter we point out some important PSCs capacitive features, aiming at providing useful information for the interpretation of capacitance measurements in relation to the validity of the MS analysis. Well-performed MS analysis will permit exploring on the influence that materials synthesis and device processing have on the operating physics parameters. A complete procedure with specific checking points is provided here in order to perform consistent MS analyses.

The most common and established method for the capacitance determination is trough impedance spectroscopy technique. ${ }^{22}$ It basically consists of applying an AC potential small perturbation $\widetilde{V}$ at a given angular frequency $\omega$, and then sensing the AC current 
response $\widetilde{I}$ of the sample. Subsequently, the impedance is obtained from as $Z^{*}=\widetilde{V} / \widetilde{I}$, being the capacitance spectrum the real part of the complex capacitance $C^{*}=1 / i \omega Z^{*}$, with $i=\sqrt{-1}$. The capacitance is typically displayed as a function of the measuring frequency $f=\omega / 2 \pi$ for several DC applied forward bias in the dark at room temperature (Figure 1). For subsequent $C$ - $V$ and MS analysis a certaín frequency has to be selected.

Several solar cell structures have been analyzed comprising planar $\mathrm{TiO}_{2}$ layers (with and without $\mathrm{TiO}_{2}$ mesoporous scaffold) as electron selective layer, and spiro-OMeTAD as hole selective material. Details on device processing, absorber compounds $\mathrm{CH}_{3} \mathrm{NH}_{3} \mathrm{PbI}_{3}$ and $\mathrm{CH}_{3} \mathrm{NH}_{3} \mathrm{PbI}_{3-x} \mathrm{Cl}_{x}$, and photovoltaic response can be seen as supplementary material. Experiments were carried out at room temperature, within an electromagnetically shielded box, in the air under $20-35 \%$ of relative humidity. No special device encapsulation was used. As it has been previously described, ${ }^{23}$ the capacitance spectrum of perovskite solar cells presents three clear regions that have been associated to different mechanisms. At low frequencies a capacitance excess arises caused by electrode polarization phenomena $C_{\mathrm{S}}$. At higher frequencies the effect of series resistances $R_{\mathrm{S}}$ is observed reducing the capacitive response. Between these two limits, a rather constant capacitance plateau appears, in principle governed by the polarizability of the material, giving rise to the geometrical capacitance per unit area $C_{g}$ at zero and negative bias. This central region may contain additional contributions in relation to the depletion layer capacitance $C_{\mathrm{dl}}$. These three capacitive features are visible at different/applied bias. However it is clear from Figure 1 that at low frequencies the capacitance is exponentially enhanced by orders of magnitude. ${ }^{24}$ On the contrary, the central capacitive plateau only increases considerably above certain DC bias (approximately $0.5 \mathrm{~V}$ ). Concerning the low frequency capacitance, it has been suggested to be associated with ionic accumulation mechanisms, mainly located at the electrode interfaces, and further correlated with the anomalous slow dynamic current transients and the current-voltage curve hysteresis. ${ }^{23-25}$ 


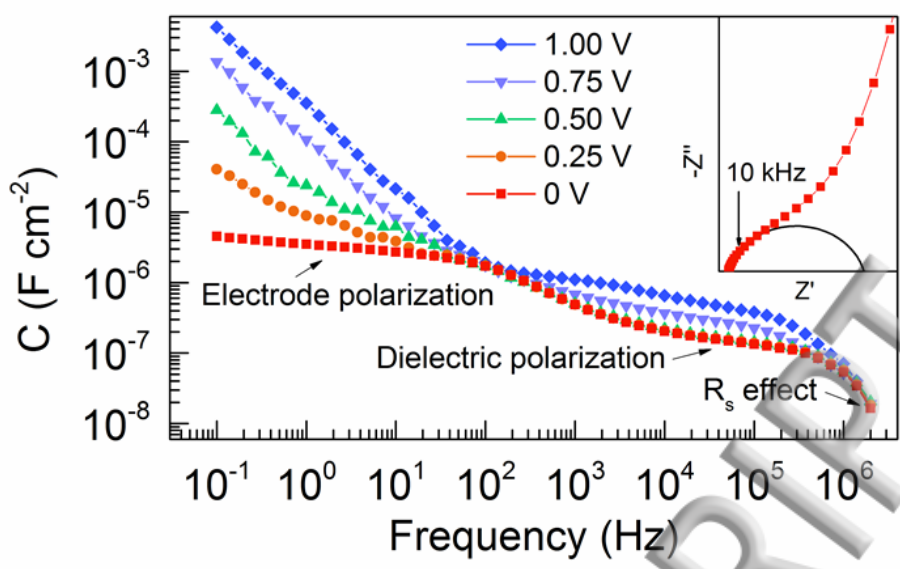

FIG. 1 Capacitance spectrum of a $\mathrm{CH}_{3} \mathrm{NH}_{3} \mathrm{PbI}_{3}$-based $\mathrm{PSC}$ (with mesoporous $\mathrm{TiO}_{2}$ matrix) at several applied DC forward bias in the dark at room temperature. Inset: impedance plot at zero bias signaling a single $R C$ arc (black solid line) fitting the high-frequency range. MS analysis sampling frequency is selected where the arc fits well the data (dielectric polarization plateau in the Bode plot). The amplitude of the AC signal was of $10 \mathrm{mV}$ (see experimental details and solar cell structure as supplementary material).

It is apparent then that in PSCs several capacitive mechanisms simultaneously operate, dominating at different frequency ranges. This means that it is not straightforward to select a standard frequency at which to perform MS analyses. In our experience the frequency domain of the central dielectric polarization region lies around 1-100 kHz, but still varies with the measurement conditions as well as with the specific characteristics of the sample.

Since a typical current rectifying behavior takes place in PSCs, it is appealing to use the theory of semiconductor heterojunctions, ${ }^{8}$ where the contact of acceptor (considering $p$ type perovskite) and donor (ETM) layers form a space-charge zone (depletion layer) that holds the barrier by equalizing the Fermi levels. In the one-side abrupt step junction approximation (the simplest case), the depletion zone width covers a prominent portion of the perovskite layer thickness, where the defect density $N$ entails a homogeneous charge distribution $\rho=q N$ [see inset in Figure 2(a)], being $q$ the elementary charge. The depletion layer capacitance per unit area can be approximated as a function of the applied bias $V$ as ${ }^{8,19}$

$$
C_{\mathrm{dl}}=\sqrt{\frac{q \varepsilon \varepsilon_{0} N}{2\left(V_{\mathrm{bi}}-V\right)}}
$$

where $\varepsilon_{0}$ is the vacuum permittivity and $\varepsilon$ is the relative dielectric constant of the perovskite material. As noted Equation 1 follows a parabolic dependence on applied 
voltage, and it implies the common relation $C_{\mathrm{dl}}{ }^{-2}=2\left(V_{\mathrm{bi}}-V\right) / q \varepsilon \varepsilon_{0} N$ used in analyzing MS plots for $V<V_{b i}$ (far from the depletion layer collapse, or flat band condition). Depletion layer width $w$ in perovskite solar cells has not only been extracted from capacitance analysis but also from direct nanometric profiling of the electrical field using Kelvin probe force microscopy. ${ }^{19,26} \mathrm{MS}$ analysis is meaningful only when $C_{\mathrm{dl}}$ is clearly identified.

In Figure 2(a) a solar cell comprising $\mathrm{CH}_{3} \mathrm{NH}_{3} \mathrm{PbI}_{3-x} \mathrm{Cl}_{x}$ as absorber in a mesoporous device structure is used as example. It allows illustrating the Mott-Schottky plot and the linear fit derived from Equation 1, which permit $N$ and $V_{\mathrm{bi}}$.calculation. Usually, reported values lie around $N \approx 10^{17} \mathrm{~cm}^{-3}$ and $V_{\mathrm{bi}} \approx 1.0 \mathrm{~V}$, in good agreement with the energetic offset between perovskite and $\mathrm{TiO}_{2}$ Fermi levels. ${ }^{17,19}$ The capacitance is extracted from the capacitance spectra at a given frequency $(10 \mathrm{kHz})$ within the highfrequency plateau linked to the dielectric response of the absorber layer. Here it should be noted that such calculation depends on the right estimation of $\varepsilon$, which exhibits significant scattering (20-35 for $\left.\mathrm{MAPbI}_{3}\right)$ in the literature. ${ }^{6,24,27,28}$ This estimation is often based on the classical parallel plate capacitor $C_{g}=\varepsilon_{0} \varepsilon / d$, being $d$ the layer thickness. As noted previously, $c_{g}$ can be readily extracted from the capacitance response at zero or negative bias at frequencies within the dielectric plateau. A selection of a low sampling frequency for the measurement of $C_{g}$ may result in the overestimation of $\varepsilon$. In order to warrant the dielectric character of $C_{g}$, it is useful to check different surface areas and/or sample thicknesses .

Figure 2(a) allows noticing that the aforementioned linear trend in $C^{-2}(V)$ (derived from Equation 1) is only present in a limited range of applied bias (low forward bias) at which $C_{\mathrm{dl}}$.is definitely identified. At reverse bias a sort of saturation deviates from the linear trend. This signals the occurrence of full depletion conditions, in contrast to other photovoltaic/technologies such as those based on $\mathrm{Si}^{29,30}$ or $\mathrm{CdTe}^{31,32}$ where the MottSchottky behavior is mainly explored in the reverse bias regimen because of wider thicknesses. The full depletion take place when $C_{\mathrm{dl}}$ equalizes $C_{g}$ (depletion layer limit), at a given reverse bias. On the other hand, MS plot deviates from the linear dependence as bias approaches the built-in voltage in the forward direction. This trend is associated to an exponential increase of the capacitance of the form $C \propto \exp \left(q V / n k_{\mathrm{B}} T\right)$, which can be better seen in the logarithm scaled right axes of Figures $2(\mathrm{a}, \mathrm{b})$ for $V>0.6 \mathrm{~V}$ [see also Figures $\mathrm{S} 1$ and $\mathrm{S} 2(\mathrm{a}, \mathrm{b}, \mathrm{e})$ ]. The exponential 
increase is a consequence of the low-frequency excess capacitance (Figure 1), denoted as $C_{\mathrm{S}}$, that even contributes to the MS plot at frequencies as high as $10 \mathrm{kHz}$ exceeding $C_{\mathrm{dl}}$. It is noted that $C_{\mathrm{s}}$ varies exponentially while $C_{\mathrm{dl}}$ exhibits a parabolic voltagedependence. In practical terms, these different dependences allow separating their effects. Several explanations have been proposed for the encountered exponential dependences. For instance the chemical capacitance, which arises from the Fermi level modulation of minority carriers, has been traditionally identified in conventional Si devices where the transition between regimens take place around $0.2 \mathrm{~V} .{ }^{8,9,33}$ Recently, charge accumulation capacitance $C_{\mathrm{S}}$ occurring at the $\mathrm{TiO}_{2} /$ perovskite interface has been proposed. $^{23,24}$ This last capacitive mechanism is able to explain the huge capacitance values reported at low frequencies (Figure 1).

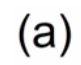

(a)
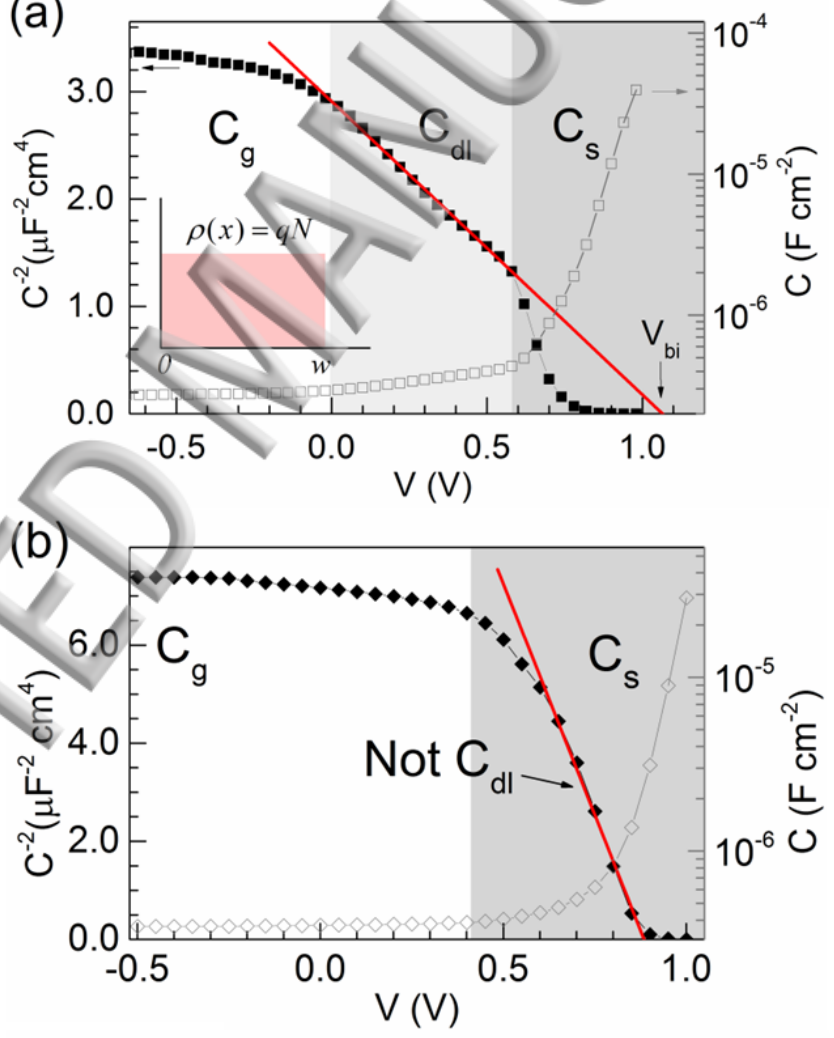

FIG. 2 Illustrative Mott-Schottky plots of PSCs (left axis, black filled dotted) and respective capacitance-voltage curves (right axis, gray open dotted). (a) $\mathrm{CH}_{3} \mathrm{NH}_{3} \mathrm{PbI}_{3-x} \mathrm{Cl}_{x}$ and (b) $\mathrm{CH}_{3} \mathrm{NH}_{3} \mathrm{PbI}_{3}$-based devices with mesoporous $\mathrm{TiO}_{2}$ matrix (see experimental section as supplementary material). While in (a) three voltage regions can be differentiated allowing for correct MS analysis, in (b) the depletion layer capacitance cannot be identified and hence MS plot analysis is not feasible. Inset in (a): the respective charge density distributions in the 
depletion layer as a function of the distance from the junction. The measurements were carried out in the dark, at room temperature and the AC perturbation was $10 \mathrm{mV}$ at $10 \mathrm{kHz}$.

The influence of capacitive term $C_{\mathrm{s}}$ on the MS representation is not always evident, as it can be seen in Figure S1, where apparently the depletion layer has just reach its collapsing limit. This has moved in some cases to disregard the effect of the parasitic $C_{\mathrm{S}}$ without examining the logarithm scaled $C-V$ curve. The clear distinction of the three voltage regions drawn in Figure 2(a) (with different capacitive mechanisms dominating in such a way that $C_{g}<C_{\mathrm{dl}}<C_{\mathrm{S}}$ ) is needed for a proper MS application. Figure 2(b) shows an example of a direct transition between $C_{g}$ and $C_{\mathrm{s}}$ without the occurrence of the depletion layer voltage-modulation in the case of $\mathrm{CH}_{3} \mathrm{NH}_{3} \mathrm{PbI}_{3}$-based solar cells including mesoporous $\mathrm{TiO}_{2}$ matrix. The last observation means that the fitting in the apparently linear region $(V>0.5 \mathrm{~V})$ is misleading as it does not stem from a true $C_{\mathrm{dl}}$ response, but from a $C_{\mathrm{s}}$-related capacitance [Figure 2(b)]. Therefore, it is then quite recommendable to endorse the MS analysis with the corresponding $C-V$ curve as presented in Figure 2. Consequently, the MS analysis can only be valid in those cases in which the distinction $C_{g}<C_{\mathrm{dl}}<\mathrm{C}_{\mathrm{s}}$ is clearly established. The reported data in Figure 2(a) evidence that our mixed-halide perovskites of the kind $\mathrm{CH}_{3} \mathrm{NH}_{3} \mathrm{PbI}_{3-x} \mathrm{Cl}_{x}$ own a large enough defect density $\left(N \approx 10^{17} \mathrm{~cm}^{-3}\right)$ so as to be detected by MS analysis, in good agreement with the assumption of $\rho=q N$. It is noted that $C_{\mathrm{dl}} \propto \sqrt{N}$ from Equation 1 . As a consequence, perovskite layers containing less defects (highly crystalline materials or with less disordered grain boundaries) cannot yield distinguishable $C_{\mathrm{dl}}$ in the MS plot representation [Figure 2(b)]. Actual defect density will depend on the preparation route and specific device processing. Moreover, MS analysis can therefore be used as a probing technique able to explore the influence of processing on defect formation.

We stress on the selection of the frequency at which the measurement is done, which must coincide with the central capacitance plateau observed at zero bias. It is evident that depending on the selected frequency, the resulting MS plot can significantly differ. We propose to select as higher measuring frequency as possible within the dielectric plateau. In our measurements a proper selection is $10 \mathrm{kHz}$ (Figure 1). It should be noted that the contact mechanisms can introduce featured capacitance responses able to completely mask $C_{\mathrm{dl}}$. In a recent impedance analysis it was found that the interfacial impedance has a rich structure that reveals different parasitic capacitive processes, serial steps for electron extraction, and a prominent inductive loop related to negative 
capacitance at intermediate frequencies. ${ }^{34}$ The application of the MS technique is clearly problematic in these cases. Alternatively, the frequency selection can rely on the Nyquist plot representation by fitting a single $R C$ arc in the high frequency range. As presented in the inset of Figure 1, the arc coincides with the experimental data at those frequencies where the dielectric polarization plateau occurs (at the Bode plot).

Furthermore, there are additional anomalous behaviors that often distort the capacitance measurements. $C-V$ analysis is always a dynamic measuring technique in which capacitance is registered in response to voltage steps. Similarly to that occurring to the current-voltage curve hysteresis, the rate and direction at which the DC applied bias is swept also produce hysteresis in the capacitance-voltage curve. As an illustration of the effect of a wide voltage window and scan rate, Figure 3 shows hysteretic capacitive measurements. In the inset of Figure 3(a) the capacitance difference $\Delta C$ between sweep directions is drawn. The presence of significant $\Delta \mathrm{C}$, and its peaks around certain specific voltages, can be understood as a product of the contact reactivity. ${ }^{34,35}$ In this case the analysis of MS slopes is absolutely meaningless as observed in the inset of Figure 3(b), producing unexpected and erroneous $V_{\mathrm{bi}} \approx 0.6-0.8 \mathrm{~V}$ estimations. $C-V$ plots at different scan rates have been previously reported for perovskite solar cells, ${ }^{35}$ and also related to degradation in the case of CdTe photovoltaic devices. ${ }^{36}$ In supplemental Figure S2(e,f), analogue to Figure 3, curves are presented for a different device architecture and polarization routines evidencing their impact on the degree of "capacitance hysteresis".

By examining supplemental Figure S1, which corresponds to the same device as that presented in Figure 2(a), one can observe how MS plots exhibit minor $\mathrm{C}_{\mathrm{s}}$ contribution when voltage scan starts at positive bias. This is also observed by comparing Fig. S2(a) and (b) that differ in the starting bias of the voltage sweep. Again positive polarization produces reproducible curves with proper MS plots [Figure S2(d)], in this case using planar devices. These last findings indicate that positive polarization pretreatment and slow scan rate are necessary conditions for correct MS analysis application. Slow scan reduces hysteretic contributions and starting positive polarization reduces interfacial capacitance accumulation. Thus it is clear that the time scale and range of DC applied bias, as well as the cell design and moving defect distribution, can distort capacitance responses. 
Also of importance is noticing the commonly observed materials instability of the PSCs, demanding a compulsory need for checking measurement reproducibility. Consequently, it is prudent to design fast and low stressing experiments on fresh cells, trying to extend the stability period of the sample. For the latter, the isolation from moisture, oxygen, UV light, high temperatures and the application of not excessive DC bias is advisable. ${ }^{37}$
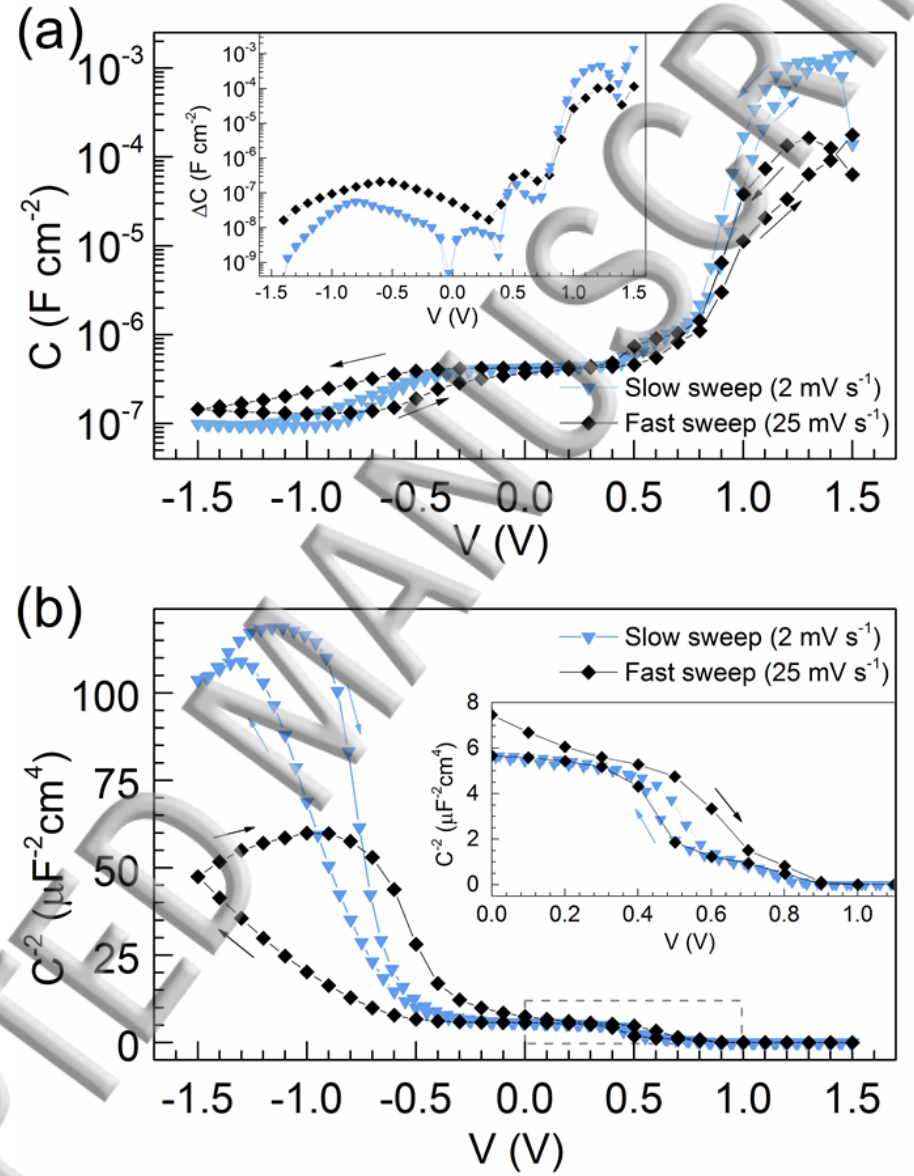

FIG. 3 (a) Capacitance-voltage curve and (b) respective Mott-Schottky plot of a $\mathrm{CH}_{3} \mathrm{NH}_{3} \mathrm{PbI}_{3}$ based PSC with mesoporous $\mathrm{TiO}_{2}$ matrix for a larger DC bias window at different scan rates, as indicated. Inset in (a) the corresponding capacitance absolute difference between scan direction $\Delta \mathrm{C}$. The inset in (b) is the typical MS plot region, pointed inside the dashed gray square. Rest of measurement conditions coincide to those of Figure 2 .

In summary, the interpretation of Mott-Schottky analysis based on measurements of capacitance for PSCs should be performed with certain precautions. Following standard 
procedures may lead to interpretation mistakes, contrary to that occurring with other photovoltaic technologies. The presence of large parasitic $C_{\mathrm{s}}$ caused by ionic polarization as well as reactivity at the contacts hinder $C_{\mathrm{dl}}$ capacitance values, and sometimes even prevents from using the MS technique. In order to accurately develop such analysis the following steps must be considered: (i) identification within the capacitance spectrum of the frequency range at which the perovskite dielectric features can be extracted. Here the dielectric constant should be estimated at zero or negative bias. (ii) Selection of a proper measuring frequency within the central plateau of the capacitance spectra. (iii) Identification of the voltage region dominated by $C_{\mathrm{dl}}$ within the $C-V$ plot, which must be checked with the MS plot for consistency. And finally (iv) verification of the sensitivity of $C-V$ analysis on scan directions, rates and pretreatment polarization. As noted previously, reactivity, predominantly at contacts, significantly alters both capacitive and resistive electrical behavior. Only under careful checking of the capacitance response, Mott-Schottky analysis can lead to meaningful and reliable parameter extraction to be properly used in device physical modeling.

\section{Supplementary Material}

Device preparation, experimental conditions and photovoltaic characteristics are detailed. Additional results on $C-K$ and Mott-Schottky analysis are also provided.

\section{Acknowledgements}

We thank financial support by Ministerio de Economía y Competitividad (MINECO) of Spain under projects (MAT2013-47192-C3-1-R and MAT2016-76892-C3-1-R), and Generalitat Valenciana (Prometeo/2014/020). O. A. acknowledges Generalitat Valenciana for a grant (GRISOLIAP2014/035). E. M.-M thanks the Ramón y Cajal program from MINECO of Spain. SCIC at UJI are also acknowledged.

\section{References}

1 H.-S. Kim, C.-R. Lee, J.-H. Im, K.-B. Lee, T. Moehl, A. Marchioro, S.-J. Moon, R. Humphry-Baker, J.-H. Yum, J. E. Moser, M. Grätzel, and N.-G. Park, Sci. Rep. 2, 591 (2012).

M. M. Lee, J. Teuscher, T. Miyasaka, T. N. Murakami, and H. J. Snaith, Science 338, 643 (2012).

M. A. Green, K. Emery, Y. Hishikawa, W. Warta, and E. D. Dunlop, Prog. Photovoltaics Res. Appl. 24, 905 (2016).

Z. Zhou, S. Pang, Z. Liu, H. Xu, and G. Cui, J. Mater. Chem. A 3, 19205 (2015).

F. Zhang, S. Wang, X. Li, and Y. Xiao, Curr. Nanosci. 12, 137 (2016).

E. J. Juarez-Perez, R. S. Sanchez, L. Badia, G. Garcia-Belmonte, Y. S. Kang, I. MoraSero, and J. Bisquert, J. Phys. Chem. Lett. 5, 2390 (2014).

I. Zarazua, J. Bisquert, and G. Garcia-Belmonte, J. Phys. Chem. Lett. 7, 525 (2016).

S. M. Sze and K. K. Ng, Physics of Semiconductor Devices, 3rd ed. (John Wiley \& Sons, Hoboken, New Jersey, 2007), p.832. 
11 This manuscript was accepted by Appl. Phys. Lett. Click here to see the version of record.

F. Fabregat-Santiago, G. Garcia-Belmonte, I. Mora-Sero, and J. Bisquert, Phys. Chem. Chem. Phys. 13, 9083 (2011).

S. S. Shin, W. S. Yang, E. J. Yeom, S. J. Lee, N. J. Jeon, Y.-C. Joo, I. J. Park, J. H. Noh, and S. I. Seok, J. Phys. Chem. Lett. 7, 1845 (2016).

W. Peng, L. Wang, B. Murali, K.-T. Ho, A. Bera, N. Cho, C.-F. Kang, V. M. Burlakov, J. Pan, L. Sinatra, C. Ma, W. Xu, D. Shi, E. Alarousu, A. Goriely, J.-H. He, O. F. Mohammed, T. Wu, and O. M. Bakr, Adv. Mater. 28, 3383 (2016).

M. Long, Z. Chen, T. Zhang, Y. Xiao, X. Zeng, J. Chen, K. Yan, and J. Xu, Nanoscale 8, 6290 (2016).

G. R. Perumallapelli, S. R. Vasa, and J. Jang, Org. Electron. 31, 142 (2016).

L. Liu, A. Mei, T. Liu, P. Jiang, Y. Sheng, L. Zhang, and H. Han, J. Am. Chem. Soc. 137, 1790 (2015).

S. Aharon, S. Gamliel, B. E. Cohen, and L. Etgar, Phys. Chem. Chem. Phys. 16, 10512 (2014).

W. Liu and Y. Zhang, J. Mater. Chem. A 2, 10244 (2014).

D. Song, P. Cui, T. Wang, D. Wei, M. Li, F. Cao, X. Yue, P. Fu, Y. Li, Y. He, B. Jiang, and M. Trevor, J. Phys. Chem. C 119, 22812 (2015).

J. Shi, J. Dong, S. Lv, Y. Xu, L. Zhu, J. Xiao, X. Xu, H. Wu, D. Li, Y. Luo, and Q. Meng, Appl. Phys. Lett. 104, 063901 (2014).

A. Guerrero, E. J. Juarez-Perez, J. Bisquert, I. Mora-Sero, and G. Garcia-Belmonte, Appl. Phys. Lett. 105, 133902 (2014).

R. Gottesman, E. Haltzi, L. Gouda, S. Tirosh, Y. Bouhadana, A. Zaban, E. Mosconi, and F. De Angelis, The Journal of Physical Chemistry Letters 5, 2662 (2014).

H. J. Snaith, A. Abate, J. M. Ball, G. E. Eperon, T. Leijtens, N. K. Noel, S. D. Stranks, J. T.-W. Wang, K. Wojciechowski, and W. Zhang, J. Phys. Chem. Lett. 5, 1511 (2014).

J. Bisquert and F. Fabregat-Santiago, in Dye-sensitized solar cells, edited by Kuppuswamy Kalyanasundaram (CRC Press, Lausanne, 2010), pp. 457.

O. Almora, I. Zarazua, E. Mas-Marza, I. Mora-Sero, J. Bisquert, and G. GarciaBelmonte, J. Phys. Chem. Lett. 6,1645 (2015).

O. Almora, A. Guerrero, and G. Garcia-Belmonte, Appl. Phys. Lett. 108, 043903 (2016).

B. Chen, M. Yang, X. Zheng, C. Wu, W. Li, Y. Yan, J. Bisquert, G. Garcia-Belmonte, K. Zhu, and S. Priya, J. Phys. Chem. Lett. 6, 4693 (2015).

C.-S. Jiang, M. Yang, Y. Zhou, B. To, S. U. Nanayakkara, J. M. Luther, W. Zhou, J. J. Berry, J. van de Lagemaat, N. P. Padture, K. Zhu, and M. M. Al-Jassim, Nat. Commun. 6, 8397 (2015).

Q. Lin, A. Armin, R. C. R. Nagiri, P. L. Burn, and P. Meredith, Nat Photon. 9, 106 (2015).

F. Brivio, K. T. Butler, A. Walsh, and M. van Schilfgaarde, Phys. Rev. B: Condens. Matter. 89, 155204 (2014).

I. Mora-Sero, G. Garcia-Belmonte, P. P. Boix, M. A. Vazquez, and J. Bisquert, Energy Environ. Sci. 2, 678 (2009).

A. F. Braña, E. Forniés, N. López, and B. J. García, J. Phys. Conf. Ser. 647, 012069 (2015).

O. Almora, L. Vaillant Roca, and A. Bosio, Rev. Cub. Fis. 31, 66 (2014).

A. E. Rakhshani, J. Appl. Phys. 90, 4265 (2001).

J. Bisquert, Nanostructured Energy Devices: Equilibrium Concepts and Kinetics. (CRC Press Taylor \& Francis Group, Boca Raton, 2014).

J. Carrillo, A. Guerrero, S. Rahimnejad, O. Almora, I. Zarazua, E. Mas-Marza, J. Bisquert, and G. Garcia-Belmonte, Adv. Energy Mater. 6, 1502246 (2016).

O. Almora, C. Aranda, I. Zarazua, A. Guerrero, and G. Garcia-Belmonte, ACS Energy Lett. 1, 209 (2016).

D. S. Albin, R. G. Dhere, S. C. Glynn, J. A. del Cueto, and W. K. Metzger, in Proc. SPIE 7412, Reliability of Photovoltaic Cells, Modules, Components, and Systems II, $74120 I$ (San Diego, 2009).

T. A. Berhe, W.-N. Su, C.-H. Chen, C.-J. Pan, J.-H. Cheng, H.-M. Chen, M.-C. Tsai, L.-Y. Chen, A. A. Dubale, and B.-J. Hwang, Energy Environ. Sci. 9, 323 (2016). 


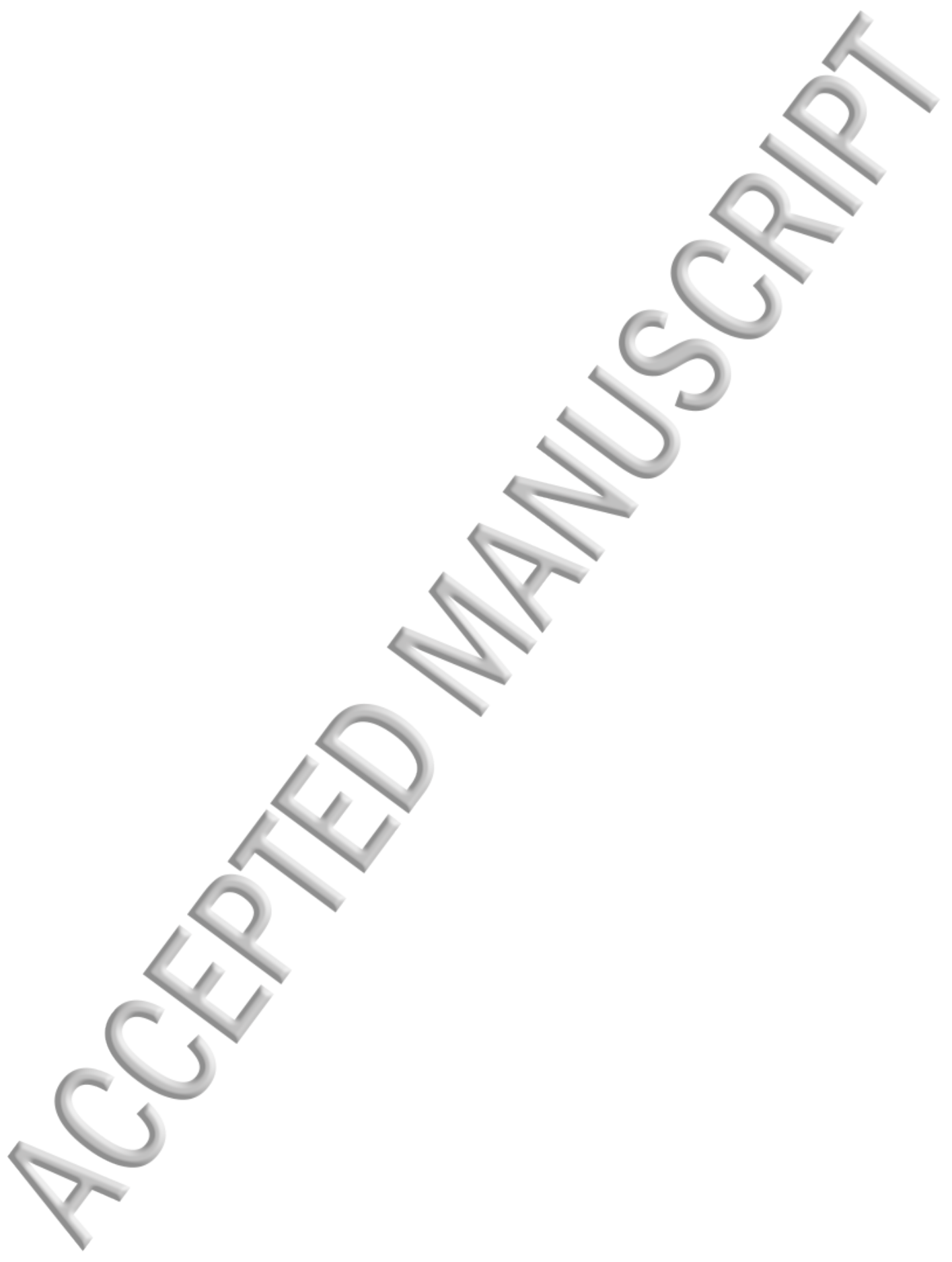


(a)

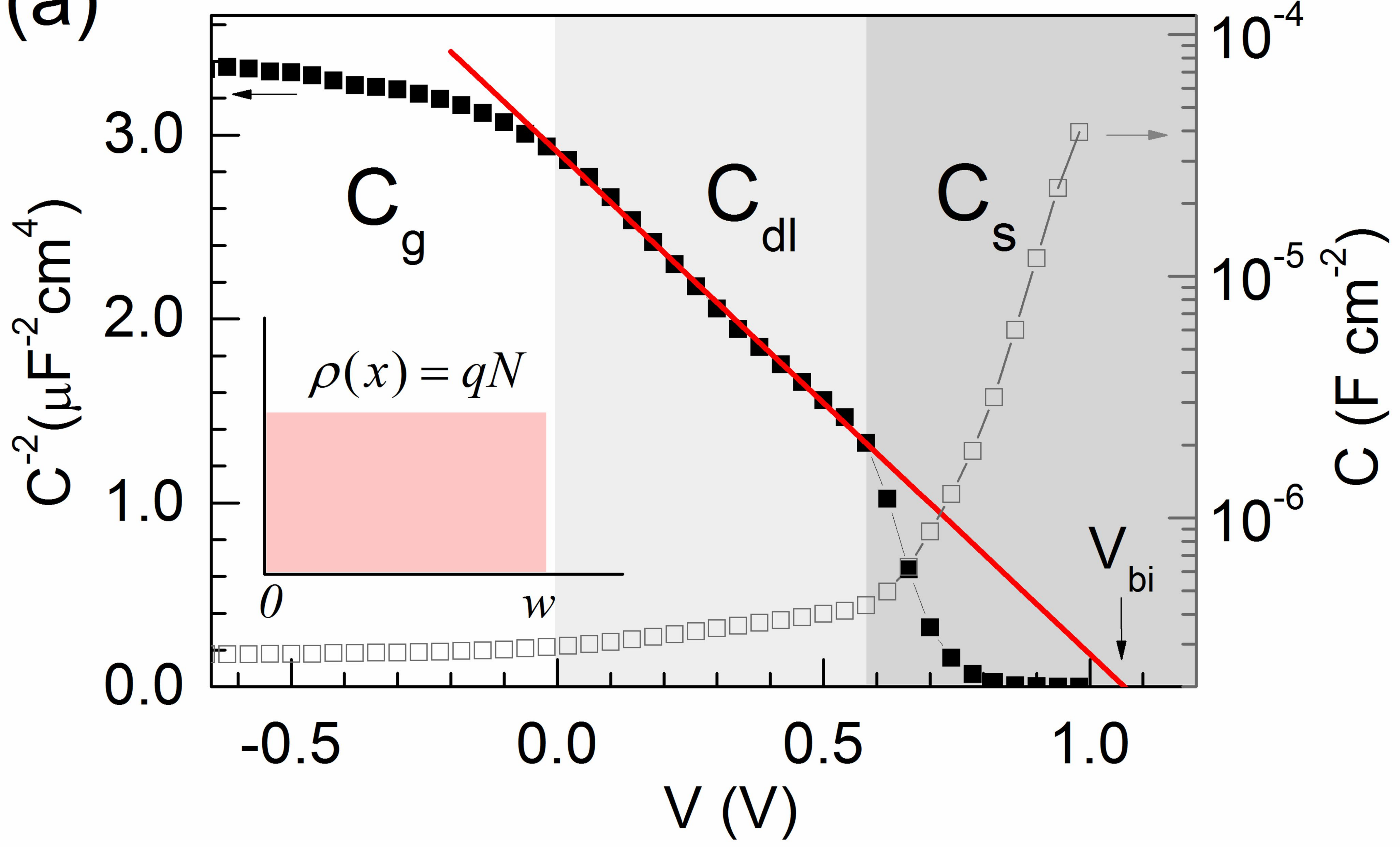

(b)

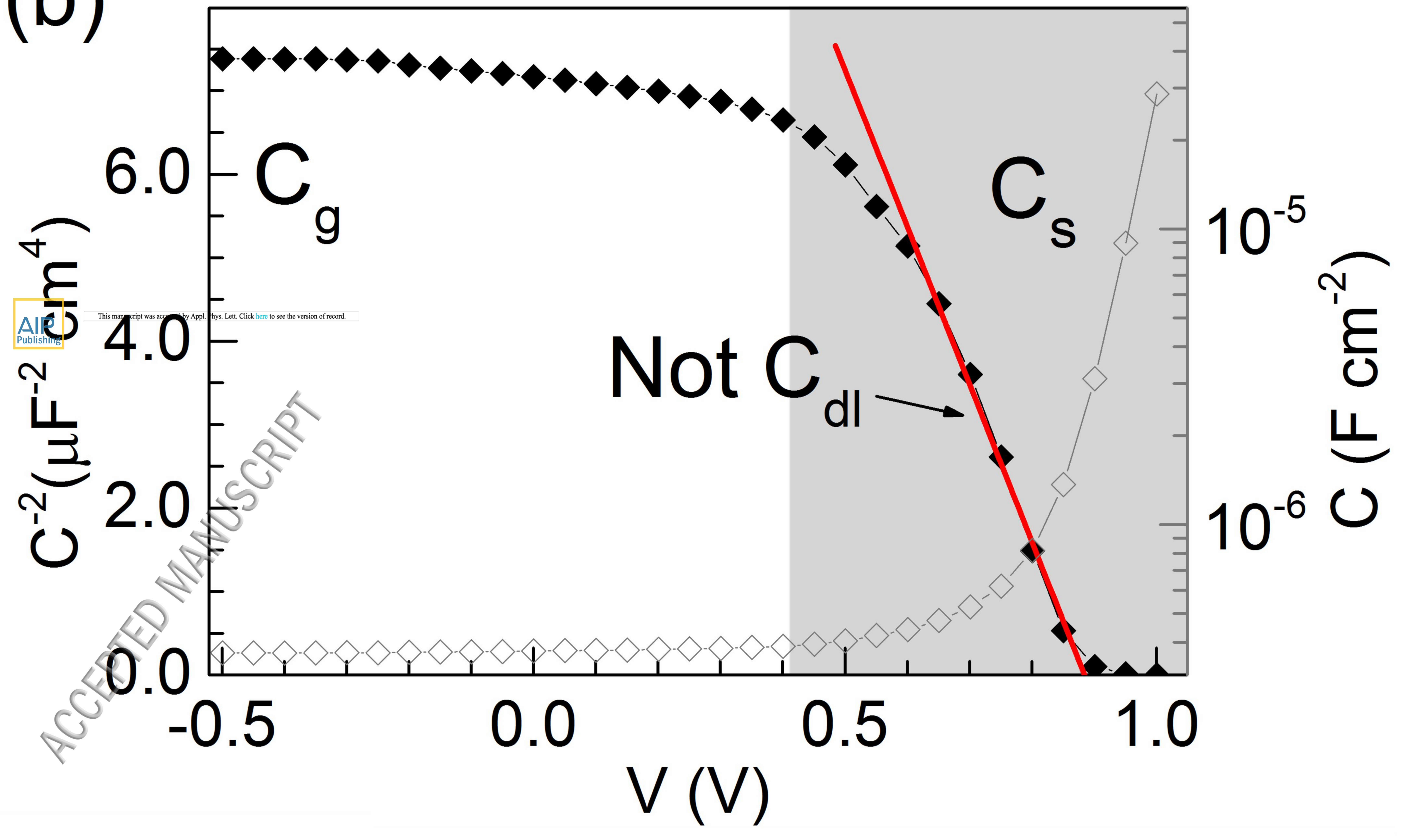


